\title{
Video Article \\ Reactive Inkjet Printing and Propulsion Analysis of Silk-based Self-propelled Micro-stirrers
}

\author{
David A. Gregory ${ }^{1}$, Piyush Kumar ${ }^{1}$, Ana Jimenez-Franco ${ }^{1}$, Yi Zhang ${ }^{1}$, Yu Zhang ${ }^{2}$, Stephen J. Ebbens ${ }^{1}$, Xiubo Zhao ${ }^{1}$ \\ ${ }^{1}$ Department of Chemical and Biological Engineering, University of Sheffield \\ ${ }^{2}$ School of Engineering, University of Liverpool
}

Correspondence to: David A. Gregory at d.a.gregory@sheffield.ac.uk, Stephen J. Ebbens at s.ebbens@sheffield.ac.uk, Xiubo Zhao at xiubo.zhao@sheffield.ac.uk

URL: https://www.jove.com/video/59030

DOI: doi:10.3791/59030

Keywords: Chemistry, Issue 146, self-propelled particles, autonomous motion, regenerated silk fibroin, catalase, hydrogen peroxide, reactive inkjet printing, catalysis, micro-stirring, micro-motors, colloids, fluid dynamics

Date Published: 4/26/2019

Citation: Gregory, D.A., Kumar, P., Jimenez-Franco, A., Zhang, Y., Zhang, Y., Ebbens, S.J., Zhao, X. Reactive Inkjet Printing and Propulsion Analysis of Silk-based Self-propelled Micro-stirrers. J. Vis. Exp. (146), e59030, doi:10.3791/59030 (2019).

\section{Abstract}

In this study, a protocol for using reactive inkjet printing to fabricate enzymatically propelled silk swimmers with well-defined shapes is reported. The resulting devices are an example of self-propelled objects capable of generating motion without external actuation and have potential applications in medicine and environmental sciences for a variety of purposes ranging from micro-stirring, targeted therapeutic delivery, to water remediation (e.g., cleaning oil spills). This method employs reactive inkjet printing to generate well-defined small-scale solid silk structures by converting water soluble regenerated silk fibroin (silk I) to insoluble silk fibroin (silk II). These structures are also selectively doped in specific regions with the enzyme catalase in order to produce motion via bubble generation and detachment. The number of layers printed determines the three-dimensional (3D) structure of the device, and so here the effect of this parameter on the propulsive trajectories is reported. The results demonstrate the ability to tune the motion by varying the dimensions of the printed structures.

\section{Video Link}

The video component of this article can be found at https://www.jove.com/video/59030/

Introduction

Artificial self-propelled micro-stirrers (SPMSs) employ a variety of propulsion mechanisms to produce motion, which can be categorized as either chemical propulsion ${ }^{1,2,3,4,5,6}$ or physical external propulsion. A common chemical propulsion mechanism is to use catalytic or enzymatic activity to either generate motion producing gradients or generate bubbles that impart momentum to the object when they detach. Previous studies have investigated several catalytic and chemical SPMSs, including polystyrene beads with platinum nanoparticles and chromium adsorbed on the surface ${ }^{1}$, gold-platinum bimetallic Janus nano-rods ${ }^{2}$, magnesium Janus micro-stirrers ${ }^{3}$, micro-stirrers made of a magnesium core and titanium dioxide shell with embedded gold nanoparticles ${ }^{4}$, and silk fibroin Janus micro-rockets with catalase embedded within the scaffold ${ }^{5}$. Physical propulsion mechanisms include magnetic ${ }^{7,8}$, optical ${ }^{9}$, and ultrasonic ${ }^{10}$ propulsion systems, all being controlled by an external physical source. Depending on the intended application, SPMS size can range from a few nanometers to several hundreds of micrometers. Examples of potential applications of these abovementioned and other SPMSs are medical diagnosis of diseases with lab-on-a-chip devices ${ }^{11}$, loading and in vivo targeted delivery of therapeutics ${ }^{12}$, environmental remediation ${ }^{3}$ (e.g., cleaning oil spills), and photocatalytic degradation of chemical and biological warfare agents, such as Bacillus anthracis and nerve agents ${ }^{4}$. Target application dependent, it is therefore desirable to be able to produce SPMSs that undergo specific trajectories such as long linear trajectories for transport challenges or rotational trajectories for micro mixing applications. The focus here is on rotational motion for stirring applications.

There is no single established method to fabricate SPMSs, but for medical and environmental applications, it is essential to use a material that is biocompatible, biodegradable, eco-friendly, readily available, cheap and allows easy fabrication of complex SPMSs without requiring sophisticated equipment. Regenerated silk fibroin (RSF) is one such material that fulfils all these parameters along with being also approved by the Food and Drug Administration (FDA).

Silk is a generic term used for several naturally occurring fibrous proteins, of which, the most commonly known is the one made by the larvae of silk moth, Bombyx mori, before its pupation. These cocoons are made of fibroin, a fibrous protein, stuck together with another protein called sericin. Silk fibroin (SF) has been found to have excellent mechanical properties, biocompatibility, and biodegradability ${ }^{13}$, which makes it an ideal choice for fabricating SPMSs. SF exists in three polymorphic forms, namely, silk I, II, and III. Silk I is a water-soluble metastable form containing mainly helixes and random coils; silk II is a water-insoluble form containing mainly antiparallel $\beta$ sheets of crystallized silk; and silk III is a threefold polyglycine II helical structure that exists at the water-air interface of the silk solution. Similar to other fibrous proteins, SF has repeating units of amino acid sequences. The naturally occurring SF of a cocoon consists of three main hexapeptide domains of such repeating 
units (GAGAGX), while X can be A, S, or Y. Through hydrogen binding, the (GA)n motifs from antiparallel $\beta$ sheet structures further stack through van der Waals forces and form hydrophobic nano crystallizations ${ }^{14,15}$.

Biocompatibility can be further improved by seeking to drive propulsion using naturally occurring enzymes to generate a concentration gradient or gas bubbles in a liquid medium (fuel). Consequently, in this study, the enzyme catalase is used as the "engine" to generate propulsion with hydrogen peroxide being used as the aqueous fuel medium. Catalase is an enzyme found in almost all living organisms. It catalyzes the decomposition of hydrogen peroxide $\left(\mathrm{H}_{2} \mathrm{O}_{2}\right)$ into water and oxygen ${ }^{16}$. The release of oxygen bubbles from the enzyme sites of the SPMSs generates a force onto the object causing it to undergo propulsion in the opposite direction of the bubble release ${ }^{5}$ (Figure 1).

In a catalytically driven SPMS, different positioning of the catalytic site results in different propulsion behaviour and trajectories ${ }^{1}$. In pursuit of generating efficient micro-stirrers, it is therefore, necessary to fabricate stirrers with well-defined geometric shapes and engine positions and compare the different powers of the engine. Here, to facilitate these investigations, it is described how regenerated silk fibroin SPMSs are fabricated at millimeter scale using reactive inkjet printing (RIJ) technology. Inkjet printing is a non-contact method for the deposition of materials. This makes the fabrication of small complex structures with high precision in generating various shapes straightforward. RIJ takes place when two or more different reacting materials are deposited and react on the substrate to produce the desired product material. Therefore, SPMSs printed with one catalytic stirrer site off center gives the object asymmetry that results in rotational motion. This approach also makes it simple to manufacture micro-stirrers in a variety of shapes and design configurations defined by computer-aided design (CAD), thus allowing easier and more accurate controllability on the desired movement during practical applications. Finally, the ability of printing devices with varying thickness that exhibit different propulsion properties is demonstrated.

This study provides a blueprint for manufacturing SPMSs with RSF at micrometer to millimeter scale. The use of RIJ technology for manufacturing RSF micro-stirrers opens the door for the highly versatile production of micro-stirrers from materials such as in situ produced scaffolds or hydrogels, which are not otherwise able to be deposited or fabricated through other means such as evaporation. After appropriate further functionalization (e.g., enzymes), these SPMSs can be suitable for environmental remediation ${ }^{3}$, such as cleaning biological toxins, organic pollutants, and chemical and biological warfare agents ${ }^{4}$.

\section{Protocol}

CAUTION: Please consult relevant material safety datasheets before use of hydrogen peroxide, sodium carbonate, ethanol, calcium chloride, and methanol. Ensure to wear all appropriate personal protective equipment including engineering controls while handling chemicals used in this protocol.

\section{Extraction of fibroin}

1. Cut $5 \mathrm{~g}$ of cleaned silk cocoons into $\sim 1 \mathrm{~cm}^{2}$ small pieces using scissors.

2. Boil $2 \mathrm{~L}$ of deionized (DI) water in a $2 \mathrm{~L}$ beaker on a magnetic hot plate under an extraction hood.

3. Add $4.24 \mathrm{~g}$ of sodium carbonate gradually and slowly into the boiling water to avoid boiling over and let it dissolve with the help of a magnetic stir bar.

4. Wait until the solution starts to boil again and add the cut pieces of cocoons into the solution. Ensure that all the silk is submerged in the solution and keep the solution boiling under constant stirring for $90 \mathrm{~min}$. Cover the beaker lightly with aluminium foil and top up with preheated DI water regularly to replenish water loss due to evaporation.

\section{Drying of fibroin}

1. Remove the extracted fibroin fibers from the sodium carbonate solution with a glass rod or spatula and wash $3 x$ with $1 \mathrm{~L}$ of preheated $\mathrm{DI}$ water for each wash, gradually decreasing the temperature for every washing step (approximately $60^{\circ} \mathrm{C}, 40^{\circ} \mathrm{C}$, and room temperature, 25 $\left.{ }^{\circ} \mathrm{C}\right)$.

2. Spread out the fibroin fibers on a $750 \mathrm{~mL}$ borosilicate glass crystallizing dish and place it into a drying oven at $60{ }^{\circ} \mathrm{C}$ under atmospheric pressure and leave to dry overnight. Once dried, store the fibroin in a closed container at room temperature.

\section{Dissolution of fibroin}

1. Prepare a ternary solution (Ajisawa's reagent) containing $4.8 \mathrm{~g}$ of $\mathrm{DI}$ water, $3.7 \mathrm{~g}$ of ethanol, and $3.1 \mathrm{~g}$ of calcium chloride ${ }^{17}$.

2. Place a two-neck round bottom flask $(100 \mathrm{~mL})$ into a water bath, made by filling a $750 \mathrm{~mL}$ borosilicate glass crystallizing dish with $600 \mathrm{~mL}$ of DI water, on top of a magnetic hot plate. Place the ternary solution inside the flask.

3. Place a thermometer in one of the necks to monitor the solution temperature accurately. Cover the other neck with aluminium foil to prevent drying out of the solution due to evaporation (or use a water-cooled reflux condenser). Heat up the solution to $80^{\circ} \mathrm{C}$. NOTE: Ensure that the bulb of the thermometer is inside the solution.

4. When the temperature of the solution is stable at $80^{\circ} \mathrm{C}$, remove the aluminium foil and add $1 \mathrm{~g}$ of dried fibroin to the solution. Add a small magnetic stir bar to ensure that the solution is mixed well throughout the dissolution process. Cover the second neck again with aluminium foil to minimize evaporation but keep the system open. Leave to dissolve for $90 \mathrm{~min}$.

\section{Dialysis of fibroin solution}

1. After $90 \mathrm{~min}$ of dissolution, leave the fibroin solution for $10 \mathrm{~min}$ to cool down to room temperature.

2. Take one $15 \mathrm{~cm}$ long dialysis tube (molecular weight cut-off $12,000-14,000 \mathrm{kDa}$ ) and tie a knot in one of the two ends. Wash it for a few minutes with running DI water from the tap. 
3. Open the other end and pour the fibroin solution inside. Using a metal clamp, close the other end of the dialysis tube ensuring that the tube is closed as tightly as possible. Attach one of the ends of the dialysis tube via a screw cap to an empty $30 \mathrm{~mL}$ plastic vial to allow the dialysis tube to float in the water.

4. Fill a $2 \mathrm{~L}$ beaker with $2 \mathrm{~L}$ of $\mathrm{DI}$ water and place the dialysis tube inside it. Change the water at regular intervals. Check the conductivity of the water every time it is changed to follow the dialysis process. The dialysis step finishes once the conductivity of the water is below $10 \mu \mathrm{S} / \mathrm{cm}$. NOTE: This process usually takes around $24-36 \mathrm{~h}$ with 5 changes of water.

5. After dialysis is complete, cut one end of the dialysis tube with scissors and pour the solution into a series of $1.5 \mathrm{~mL}$ tubes. Then, centrifuge for $5 \mathrm{~min}$ at $16,000 \times \mathrm{g}$ to remove any particles inside the fibroin solution. Collect the supernatant in a $30 \mathrm{~mL}$ plastic vial and store it at $4{ }^{\circ} \mathrm{C}$.

\section{Determination of RSF solution concentration}

1. Weigh a clean glass slide $\left(\mathrm{W}_{1}\right)$. Add $200 \mu \mathrm{L}$ of silk solution $\left(\mathrm{V}_{1}\right)$.

2. Leave the glass slide in an oven at $60^{\circ} \mathrm{C}$ for $2 \mathrm{~h}$.

3. Weigh the glass slide again $\left(\mathrm{W}_{2}\right)$.

4. Calculate the concentration of the silk solution $(w / v)$ using the following formula:

$$
\operatorname{Silk}(w / v)=\frac{W_{2}-W_{1}}{V_{1}}
$$

\section{Preparation of inks for printing}

1. Prepare ink $A$ (final volume $1.5 \mathrm{~mL}$ ) by mixing the fibroin solution $(40 \mathrm{mg} / \mathrm{mL})$, polyethylene glycol $400\left(P E G_{400} ; 14 \mathrm{mg} / \mathrm{mL}\right)$ and deionized water for printing the main body of the SPMSs.

2. For printing the catalytic engine of the SPMSs, mix fibroin $(40 \mathrm{mg} / \mathrm{mL}), \mathrm{PEG}_{400}(12 \mathrm{mg} / \mathrm{mL})$, catalase $(6 \mathrm{mg} / \mathrm{mL}$ with catalytic activity of $>20,000$ units $/ \mathrm{mg}$ ) and deionized water to make $1.5 \mathrm{~mL}$ of ink B.

3. Prepare $1.5 \mathrm{~mL}$ of ink $\mathrm{C}$ by dissolving Coomassie brilliant blue $(0.05 \mathrm{mg} / \mathrm{mL})$ in methanol. NOTE: Methanol is used for converting the fibroin random coils to rigid beta sheets by printing ink $C$ on top of ink $A$ or ink $B$. Coomassie brilliant blue is used to provide a contrasting color of SPMSs to aid auto tracking of the SPMSs during propulsion.

\section{Reactive inkjet 3D printing}

NOTE: The inkjet printer used in these experiments is based on piezo actuated jetting devices with glass nozzles. There are several commercially available inkjet printers for research that can duplicate these functions.

1. Use jetting devices with $80 \mu \mathrm{m}$ nozzle diameter for printing the inks on a silicon substrate placed on the stage at a working distance between the nozzle and the Si-wafer substrate of around $5 \mathrm{~mm}$. The geometric shapes of the SPMSs are digitally defined as a series of points of X-Y coordinates in a spreadsheet file.

NOTE: The printer reads the coordinates serially and runs the printer accordingly. Each coordinate point makes the printer jet once through the jetting device. Separate spreadsheet files are created for inks A and B (see Supplemental Files [SPMS Main Body.xlsx and SPMS Engine.xlsx])

2. Load the three inks (A, B, and $C)$ into three reservoirs $(1.5 \mathrm{~mL}$ each) and then adjust the backpressure using the backpressure valve for each individual channel to ensure that the ink is not dripping from the jetting devices. NOTE: Three jetting devices on independent channels are needed.

3. Adjust the jetting parameters (rise time 1, dwell time, fall time, eco time, rise time 2, idle voltage, dwell voltage, echo voltage) for each channel to ensure that each ink gives a good stable droplet formation (Figure 2).

NOTE: These parameters are jetting device and ink dependant and will need to be adjusted accordingly.

4. Print the silk fibroin ink layer-by-layer alternating with methanol on clean polished Si-wafer substrates: stage 1, printing of ink $A$ (main body); stage 2: printing of ink C (curing ink); stage 3: printing of ink B (catalytic ink for engine sites); stage 4: printing of ink C (curing ink); stage 5: repeat stages 1-4 for desired layers required (e.g., 100).

NOTE: Two example designs for the 4 stages are included in Supplemental Files; SPMS Main Body.xlsx is used for stage 1 and stage 2 , and SPMS Engine.xlsx is used for stage 3 and stage 4

5. Print two batches of fibroin SPMSs with 200 layers and 100 layers thickness, respectively. NOTE: The catalase engine is located on the side of one end of each stirrer. Thus, the stirrers have one catalytic engine (see Figure 1 red region).

6. To remove the samples off the Si-wafers, immerse samples in DI water and gently agitate until detachment occurs.

\section{Data acquisition/tracking and trajectory analysis of self-propelled stirrers}

1. Clean a glass petri dish ( $9 \mathrm{~cm}$ in diameter) with $\mathrm{DI}$ water ensuring that the surface is dust free.

2. Once clean and dry, add $10 \mathrm{~mL}$ of pre-filtered $(0.45 \mu \mathrm{m}) 5 \% \mathrm{w} / \mathrm{v} \mathrm{H}_{2} \mathrm{O}_{2}$ into the petri dish and leave to settle. Light up the bottom of the petri dish with a cool white light-emitting diode (LED) light source and use a high-speed camera with macro zoom lens to capture the motion from above. Save videos as avi files.

NOTE: See Table of Materials for details of the equipment used.

3. Wash the printed silk stirrers for 10 min by submerging them in DI water to remove any unbound $\mathrm{PEG}_{400}$. Carefully take one washed stirrer with the tip of a sterile syringe needle and place it in the center of the petri dish. When the washed stirrer touches the $\mathrm{H}_{2} \mathrm{O}_{2}$ fuel, bubbles 
start forming around the engine and circular motion of the stirrer is observed. When the system appears stable (usually $10-30 \mathrm{~s}$ later), press record in the recording software to start capturing the video.

4. Perform tracking of the micro stirrers on a frame by frame basis, tracking each end of the stirrers as indicated by points $A$ and $B$ in Figure 3 . NOTE: This can be done manually or with the aid of tracking software.

5. From the obtained tracking data, calculate the instantaneous velocity between two consecutive frames (e.g., 1 and 2 ) using the equation below and average the resulting velocities from the entire sequence to obtain the mean instantaneous velocity.

6. Further to this, calculate the angle of orientation $\varphi$. Then use the rate of change of $\varphi$ to determine the rotational velocity (Figure 3 ).

$$
v_{\text {inst }}=\frac{\sqrt{\left(x_{2}-x_{1}\right)^{2}+\left(y_{2}-y_{1}\right)^{2}}}{\Delta t_{\text {fiame }}}
$$

NOTE: When calculating instantaneous velocities from tracked image data, it is important that the initial image of an object with known dimensions is taken to be able to calculate correct pixel to micrometer values. These values will depend on the camera, objective, and distance used. Depending on the type of printed particle, choose different tracking points for calculating the velocity. For example, here tracking points $\mathrm{A}, \mathrm{B}$, and $\mathrm{C}$ (center of mass) are all used to determine instantaneous velocities (Figure 3 ).

\section{Characterization of SPMSs by SEM}

1. Remove unused and used SPMSs from the Si-wafer or bulk solution and transfer them onto $10 \mathrm{~mm}$ wide carbon sticky pads mounted onto aluminium scan electron microscopy (SEM) stubs. Dry the samples in a drying oven for $10 \mathrm{~min}$ at $60^{\circ} \mathrm{C}$.

2. Load the sample stubs onto the sputter coater stage. Sputter coat (argon plasma at 0.05 Torr) 50-100 nm of gold onto the samples, ensuring a homogeneous gold surface coverage of the sample.

3. Remove the sample stubs from the sputter coater and image in an SEM under vacuum at $5.0 \mathrm{kV}$. NOTE: Very high acceleration voltages can burn the silk and give rise to false features.

\section{Representative Results}

After boiling the silk, it is expected that the dried fibers are around one third lighter than before, indicating the successful removal of sericin.

During the dissolution of the silk in Ajisawa's reagent, the fibers should be completely dissolved, and a yellow viscous liquid should be recovered. After dialysis, the silk solution should be less viscous but still show a slightly yellow color. If the silk has turned into gel, this indicates that dissolution was not done successfully.

Stable droplets formed from the jetting devices will enable the higher definition of the printed samples. Figure 2 shows an example of a stable single droplet to give good printing results such as the printed silk-stirrers shown in Figure 4. It is normal, depending how viscous the ink is, that spreading occurs on the substrate.

Depending on the inkjet printers used and the droplet size, the distance between each printed droplet needs to be adjusted in such a way that they overlap to generate connected lines. If the droplets are too far apart, the printed structure will break up. In addition to this, if not enough layers are printed, there is a chance of the micro-stirrers breaking when placed into fuel solution. Once the stirrers have been removed off the substrate and washed, placing them into the hydrogen peroxide fuel solution should instantly result in bubbles being formed. The success rate of good bubble release depends strongly on the enzyme activity; if the enzyme activity is low, fewer bubbles will form thus leading to poor propulsion results. Figure $\mathbf{5}$ shows how the surface morphology of the stirrers is altered due to the bubbles being released from the inner structures generating small pores. A successful micro-stirrer will look similar to the ones that can be seen in Figure $\mathbf{6}$ and the two Supplemental Videos $\mathbf{S 1}$ and $\mathbf{S 2}$ respectively.

Figure 6 shows still video frames of two representative, 100-layer (Figure 6A) and 200-layer (Figure 6B) micro-stirrers in 5\% $\mathrm{H}_{2} \mathrm{O}_{2}$ fuel. The red and green lines indicate the trajectories tracked (see Supplemental Videos $\mathbf{S 1}$ and $\mathbf{S 2}$ ). The rotational velocity can be determined by the rate of change of orientation ( $\phi$, Figure 3 ) as shown in Figure 7. Comparison of 100-layer and 200-layer catalase doped micro-stirrers shows a distinctive increase in rotational velocity of $\sim 0.6$ fold from $60 \pm 6 \mathrm{rpm}$ to $100 \pm 10 \mathrm{rpm}$ (Figure 7). 


\section{$2 \mathrm{H}_{2} \mathrm{O}_{2} \quad 2 \mathrm{H}_{2} \mathrm{O}+\mathrm{O}_{2}$}
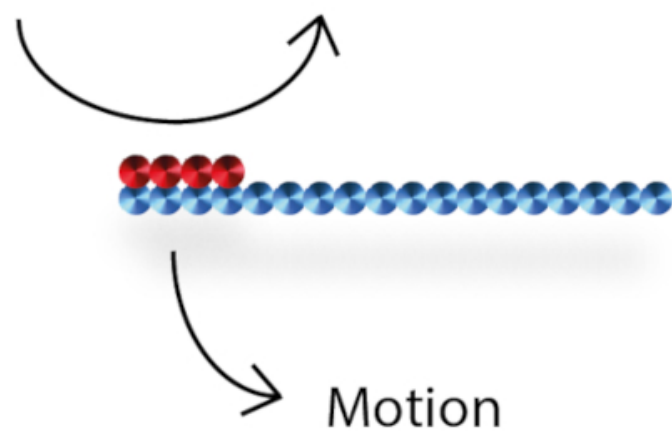

Figure 1: Schematic illustration of the catalytic breakdown of hydrogen peroxide into water and oxygen by catalase embedded in the stirrer's scaffold at desired locations (shown in red). The product oxygen bubbles provide the necessary propulsion for moving the stirrer. Please click here to view a larger version of this figure.

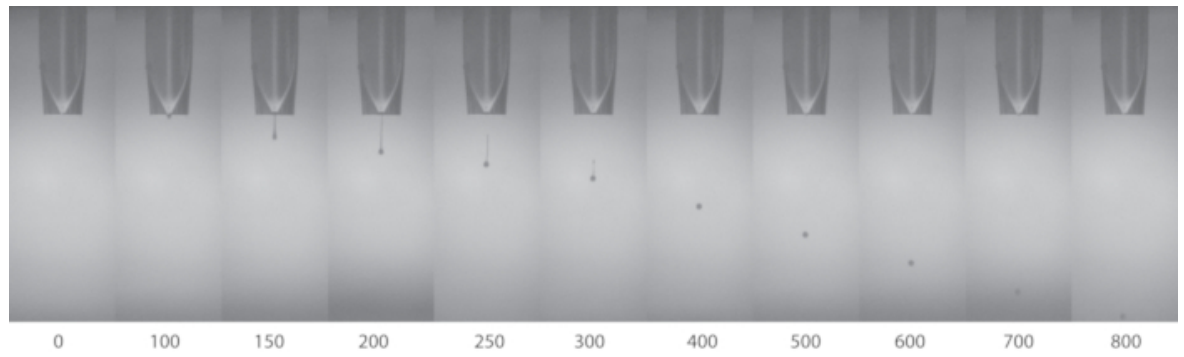

Figure 2: The time lapse images of the droplet formation of RSF from a jetting device (nozzle diameter $80 \mu \mathrm{m}$ ). The numbers below the images represent the time elapsed, in microseconds $(\mu \mathrm{s})$, since the initiation of jetting of the silk ink droplet. Please click here to view a larger version of this figure. 


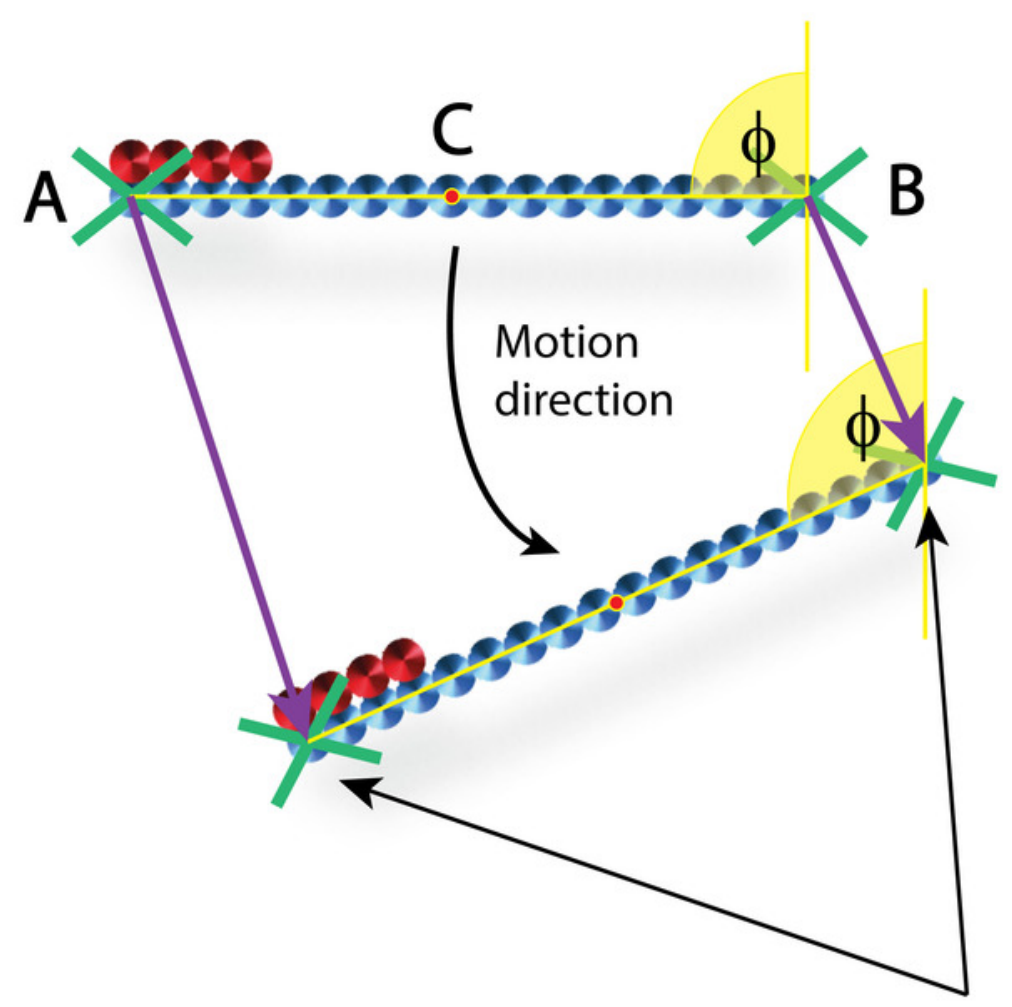

\section{Tracking points}

Figure 3: Schematic representation of particle tracking over two consecutive frames. $A$ and $B$ indicate tracking points and $C$ indicates the center of mass. $\varphi$ indicates the angle of orientation. SPMS trajectory direction is indicated by the curved black arrow. Please click here to view a larger version of this figure.
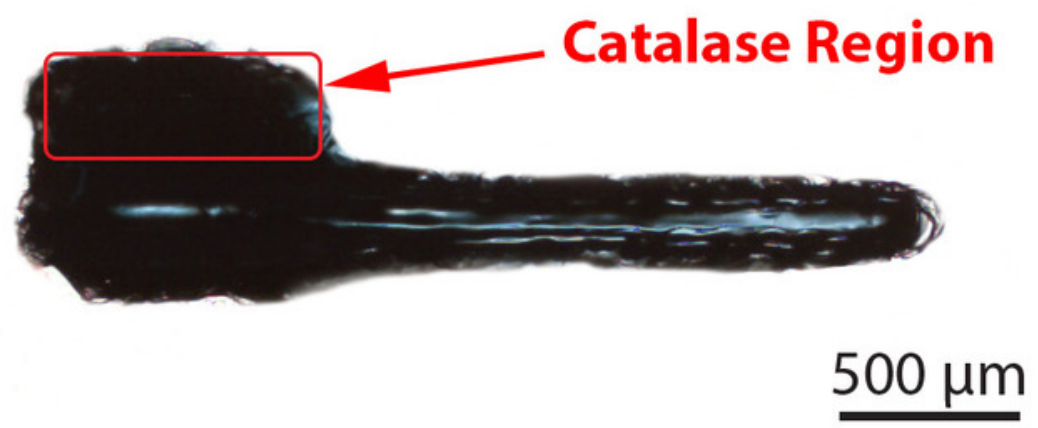

Figure 4: Light micrograph of a freshly RIJ printed micro-stirrer (100 layers) before washing. Red box denotes catalase doped region (engine region). Please click here to view a larger version of this figure. 

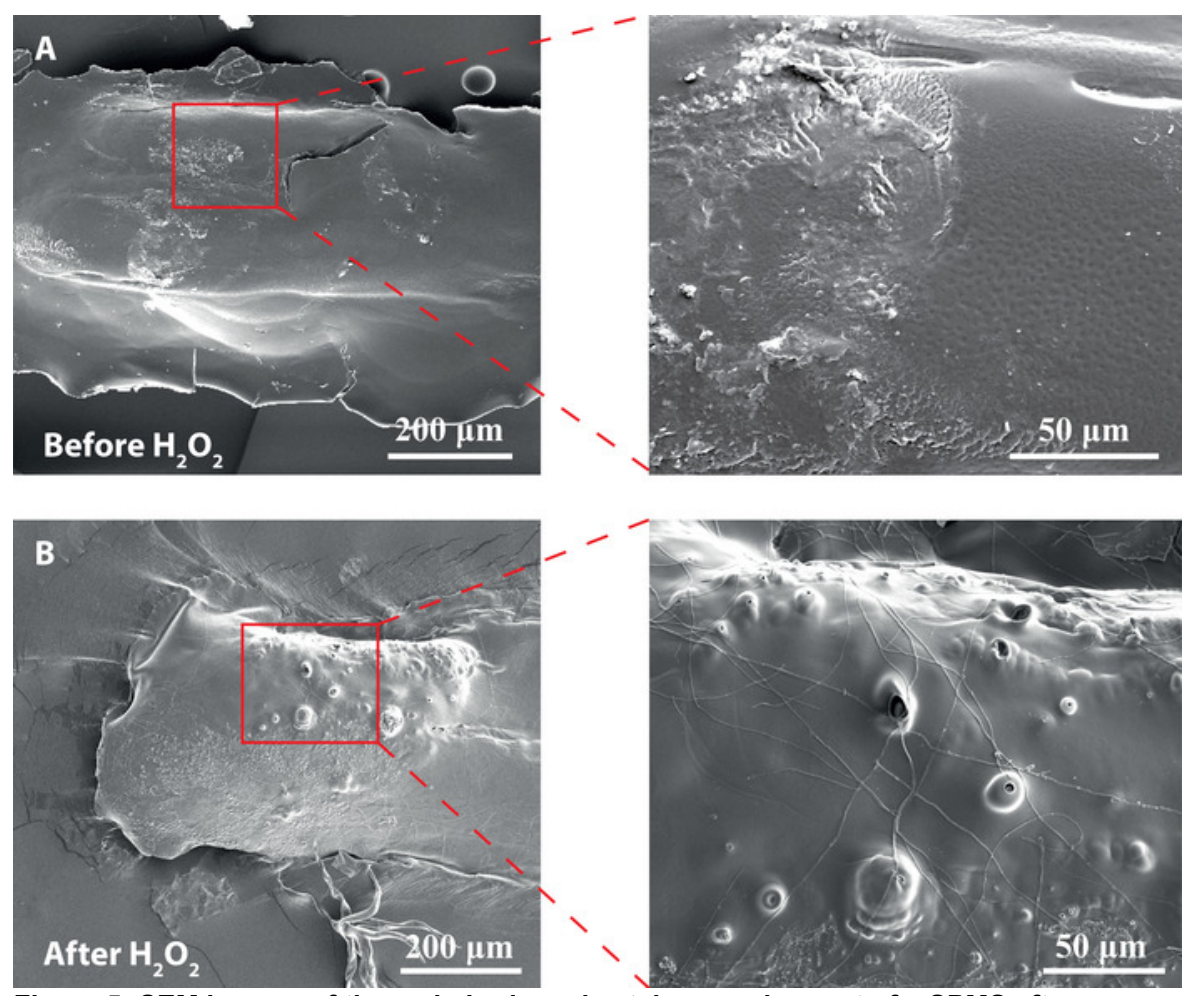

Figure 5: SEM images of the main body and catalase engine part of a SPMS after pores are formed due to bubble release. Pores can be clearly seen on the engine surface in the SEM images of the SPMSs originating from the oxygen bubble release. (A) Silk micro-stirrers before exposure to $5 \% \mathrm{w} / \mathrm{v} \mathrm{H}_{2} \mathrm{O}_{2}$ fuel solution. (B) Silk SPMS after exposure to $5 \% \mathrm{w} / \mathrm{v} \mathrm{H}_{2} \mathrm{O}_{2}$ fuel solution. Images on the right are enlargements of the red regions. Please click here to view a larger version of this figure. 

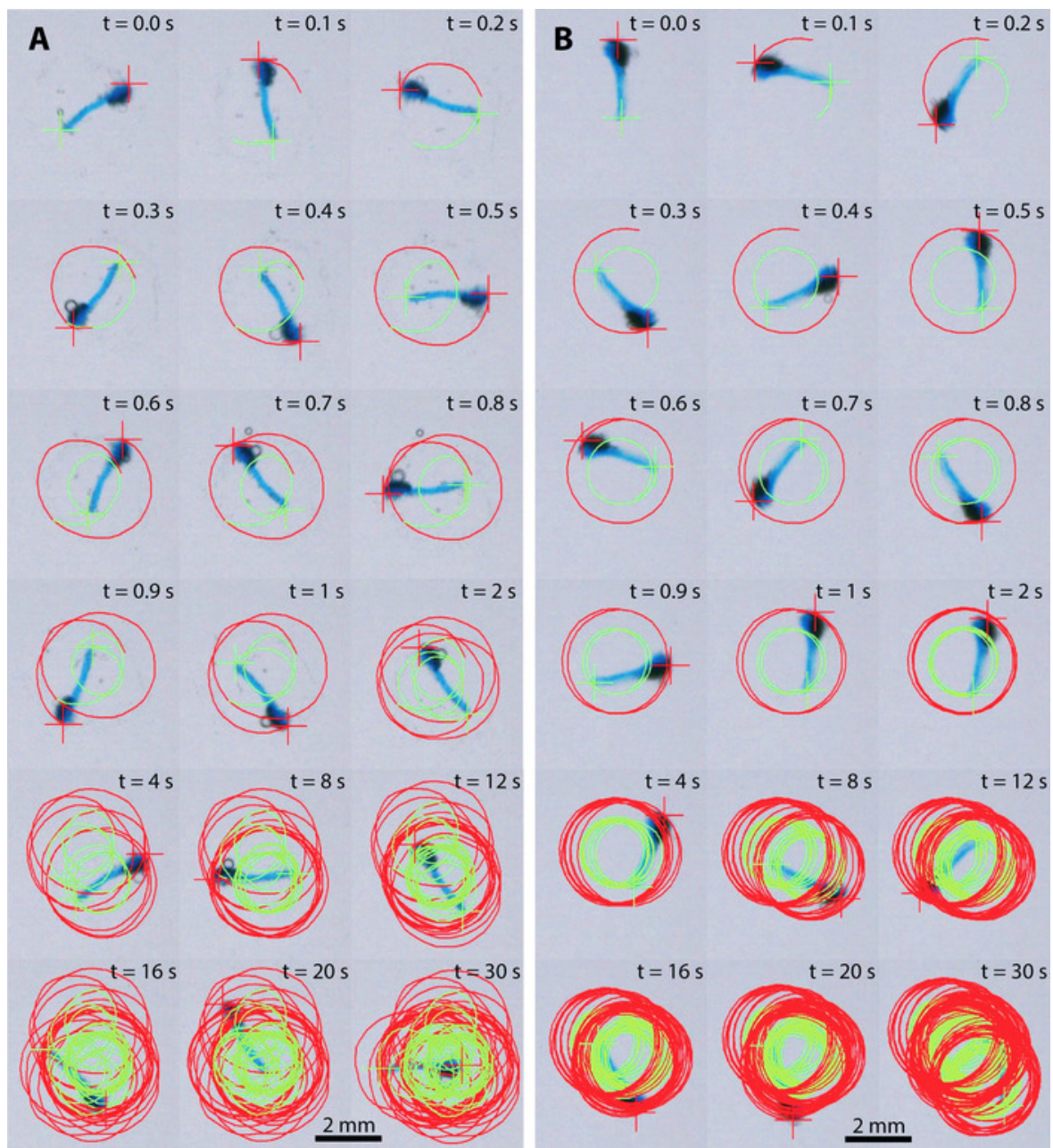

Figure 6: Video frames of two micro-stirrers in $\mathbf{5 \%}$ fuel solution showing the trajectory over time. (A) 100-layer micro-stirrers. (B) 200layer micro-stirrers. Please click here to view a larger version of this figure.
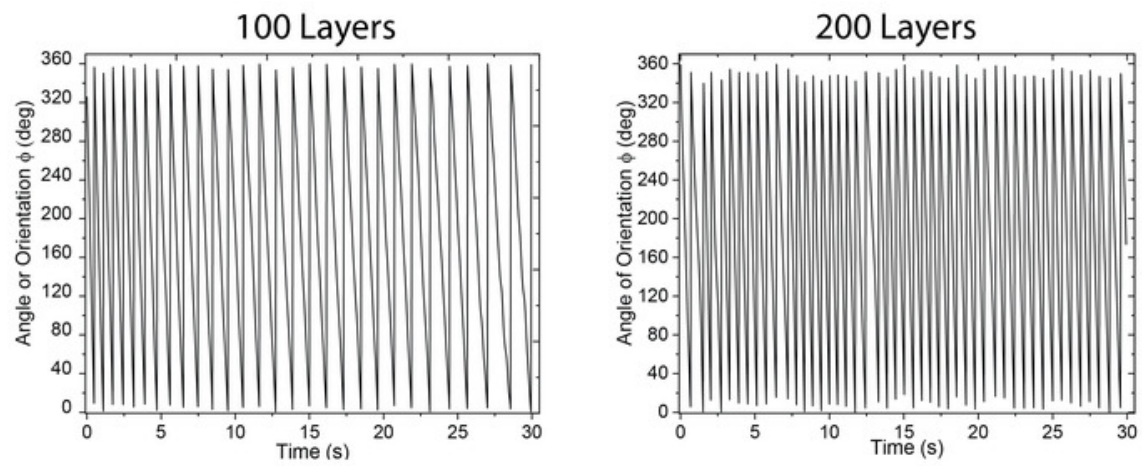

Figure 7: Comparison of angle of orientation $(\varphi)$ for 100-layer $(60 \pm 6 \mathrm{rpm})$ and 200 -layer $(100 \pm 10 \mathrm{rpm})$ micro-stirrers. Please click here to view a larger version of this figure.

Supplemental Video S1: Representative 100-layer self-motile micro-stirrer in $5 \% \mathbf{w} / \mathbf{v ~}_{\mathbf{2}} \mathrm{O}_{2}$ undergoing propulsion. Please click here to download this file.

Supplemental Video S2: Representative 200-layer self-motile micro-stirrer in $\mathbf{5} \% \mathbf{w} / \mathbf{v} \mathbf{H}_{2} \mathrm{O}_{2}$ undergoing propulsion. Please click here to download this file.

\section{Discussion}

A key feature of SPMSs produced in this protocol is the ability to quickly and easily design different shapes and structures via RIJ of silk doped with enzymes such as catalase and achieve chemically driven motion via bubble propulsion ${ }^{5}$. This together with the high biocompatibility ${ }^{18}$ of 
these stirrers makes them highly desirable for future applications for both water remediation challenges as well as for lab-on-a-chip applications for medical diagnosis devices.

Here, the propulsion ability is demonstrated by utilizing a simple line design with an engine part printed to the side of it as shown in Figure 1. The red dots represent the catalytically active catalase doped engine parts and the blue dots represent the inactive parts. To be able to generate $3 \mathrm{D}$ shapes via RIJ, it is necessary to print multiple layers in order to generate a z-axes height. Here, the numbers of layers deposited onto a Si-wafer were 100 and 200. By varying the number of layers, a difference in propulsion velocity/rotation comparable to depositing double the amount of material can be found. In order to have well-defined structures during inkjet printing, it is important that the correct jetting parameters are selected in order to achieve a well-defined droplet as shown in Figure 2. These parameters will vary according to the ink used and the jetting devices. If the ink does not produce stable droplets, then the ink is most likely no longer suitable for printing and is most likely starting to turn into gel. It is important to note that the resolution limit strongly depends on the size of nozzle used, and smaller nozzles allow for higher resolution and smaller structures/particles being printed.

An example of RIJ printed silk stirrer is shown in Figure 4, where the catalase doped engine part (as indicated by the red marked region) can be seen attached to the side of the main body (also see the schematic in Figure 1 for details). The printed silk scaffold is a material that allows for the fuel solution to diffuse throughout the entire 3D structure, and thus oxygen bubbles are formed during the decomposition of hydrogen peroxide via catalase. The oxygen bubbles being released generate micron-scale pores in the silk scaffold structure as can be seen by comparing the SEM micrographs before exposure to $\mathrm{H}_{2} \mathrm{O}_{2}$ fuel (Figure 5A) and after $\mathrm{H}_{2} \mathrm{O}_{2}$ exposure (Figure 5B). In order to ensure that motion is due to the decomposition of $\mathrm{H}_{2} \mathrm{O}_{2}$ fuel but not surface tension driven via the release of $\mathrm{PEG}_{400}$, it is important that stirrers are initially immersed in water for a period of at least $10 \mathrm{~min}$ and tested in DI water for surface tension motion prior to propulsion in fuel solution.

The use of $\mathrm{PEG}_{400}$ allows for better release of bubbles from the silk surface ${ }^{19}$ as previously explained by Gregory et al. ${ }^{5}$ but can also give rise to surface tension driven propulsion, which may be desirable depending on the application as previously described ${ }^{20}$. This second mechanism also gives the opportunity to produce SPMSs with two mechanisms that are time dependant and can be advantageous for certain applications that would for example expect initial vigorous stirring at the start followed by slower continued stirring for extended periods of time ${ }^{20}$.

In conclusion, by using RIJ to produce autonomous self-propelled devices, a vast range of shapes and sizes can be easily designed and printed. Silk as a base material for the devices gives opportunities to easily encapsulate enzymes and other moieties into the structures giving the possibility to add functions to these devices.

\section{Disclosures}

The authors have nothing to disclose.

\section{Acknowledgments}

The authors would like to acknowledge support from the EPSRC via X. Zhao's reactive inkjet printing of silk materials awards (EP/N007174/1 and EP/N023579/1), S. J. Ebbens Career Acceleration Fellowship, and Healthcare Technology Impact Fellowship (EP/J002402/1 and EP/ N033736/1). The authors also thank Dr. Qingyou Xia from the State Key Laboratory of Silkworm Genome Biology, Southwest University, China for providing silk cocoons.

\section{References}

1. Gregory, D. A., Campbell, A. I., Ebbens, S. J. Effect of Catalyst Distribution on Spherical Bubble Swimmer Trajectories. The Journal of Physical Chemistry C. 119 (27), 15339-15348 (2015)

2. Paxton, W. F. et al. Catalytic Nanomotors: Autonomous Movement of Striped Nanorods. Journal of the American Chemical Society. 126 (41), 13424-13431 (2004).

3. Gao, W. et al. Seawater-driven magnesium based Janus micromotors for environmental remediation. Nanoscale. 5 (11), $4696-4700$ (2013).

4. Li, J. et al. Water-Driven Micromotors for Rapid Photocatalytic Degradation of Biological and Chemical Warfare Agents. ACS Nano. 8 (11), 11118-11125 (2014).

5. Gregory, D. A., Zhang, Y., Smith, P. J., Zhao, X., Ebbens, S. J. Reactive Inkjet Printing of Biocompatible Enzyme Powered Silk Micro-Rockets. Small. 12 (30), 4048-4055 (2016).

6. Ismagilov, R. F., Schwartz, A., Bowden, N., Whitesides, G. M. Autonomous movement and self-assembly. Angewandte Chemie-International Edition. 41 (4), 652-654 (2002).

7. Li, T. et al. Highly Efficient Freestyle Magnetic Nanoswimmer. Nano Letters. 17 (8), 5092-5098 (2017).

8. Barbot, A., Decanini, D., Hwang, G., Controllable Roll-to-Swim motion transition of helical Nanoswimmers; in 2014 IEEE/RSJ International Conference on Intelligent Robots and Systems. 4662-4667 (2014).

9. Xuan, M. et al. Near Infrared Light-Powered Janus Mesoporous Silica Nanoparticle Motors. Journal of the American Chemical Society. 138 (20), 6492-6497 (2016).

10. Garcia-Gradilla, V. et al. Functionalized ultrasound-propelled magnetically guided nanomotors: toward practical biomedical applications. ACS Nano. 7 (10), 9232-9240 (2013).

11. Baraban, L. et al. Catalytic Janus Motors on Microfluidic Chip: Deterministic Motion for Targeted Cargo Delivery. ACS Nano. 6 (4), $3383-3389$ (2012).

12. Ghalanbor, Z., Marashi, S.-A., Ranjbar, B. Nanotechnology helps medicine: Nanoscale swimmers and their future applications. Medical Hypotheses. 65 (1), 198-199 (2005).

13. Lu, Q. et al. Water-Insoluble Silk Films with Silk I Structure. Acta Biomaterialia. 6 (4), 1380-1387 (2010).

14. Wilson, D., Valluzzi, R., Kaplan, D. Conformational Transitions in Model Silk Peptides. Biophysical Journal. 78 (5), $2690-2701$ (2000).

15. Fink, T. D., Zha, R. H. Silk and Silk-Like Supramolecular Materials. Macromolecular Rapid Communications. 39 (17), 1700834 (2018). 
16. Chelikani, P., Fita, I., Loewen, P. C. Diversity of structures and properties among catalases. Cellular and Molecular Life Sciences CMLS. 61 (2), 192-208 (2004).

17. Ajisawa, A. Dissolution of silk fibroin with calciumchloride/ethanol aqueous solution. Journal of Sericultural Science of Japan. 67 (2), $91-94$ (1998).

18. Zhang, Y., Gregory, D. A., Smith, P. J., Zhao, X., Regenerated silk fibroin as an inkjet printable biomaterial; in Printing For Fabrication (NIP). 406-409 Society for Imaging Science and Technology, Manchester (2016).

19. Gregory, D. A., Zhang, Y., Smith, P. J., Ebbens, S. J., Zhao, X., Altering the Bubble Release of Reactive Inkjet Printed Silk Micro-rockets; in Printing For Fabrication (NIP). 452-456 Society for Imaging Science and Technology, Manchester (2016).

20. Zhang, Y. et al. Reactive Inkjet Printing of Functional Silk Stirrers for Enhanced Mixing and Sensing. Small. e1804213, (2018). 